\title{
Transferability of Sorghum Genic Microsatellite Markers to Peanut
}

\author{
Siddanna B. Savadi ${ }^{1}$, Bashasab Fakrudin ${ }^{1 *}$, H. L. Nadaf $^{2}$, M. V. C. Gowda ${ }^{2}$ \\ ${ }^{1}$ Department of Biotechnology, University of Agricultural Sciences, Dharwad, India; ${ }^{2}$ Department of Genetics and Plant Breeding, \\ University of Agricultural Sciences, Dharwad, India. \\ Email: bfakrudin@gmail.com
}

Received April 14 ${ }^{\text {th }}, 2012$; revised May 12 $2^{\text {th }}, 2012$; accepted May 20 ${ }^{\text {th }}, 2012$

\begin{abstract}
Currently development of new marker types has shifted from anonymous DNA fragments to gene-based markers. Simple Sequence Repeats (SSRs) are useful DNA markers in plant genetic research including in peanut. However, de novo development of SSRs is expensive and time consuming. Gene-based DNA markers are transferable among related species owing to the conserved nature of genes. In this study transferability of sorghum EST-SSR (SbEST-SSR) markers to peanut was prospected. A set of 411 SbEST-SSR primer pairs were used to amplify peanut genomic DNA extracted from cultivated peanut where $39 \%$ of them successfully amplified. A comparison of amplification patterns between sorghum and peanut showed similar banding pattern with majority of transferable SbEST-SSRs. Among these transferable SSR markers, $14 \%$ have detected polymorphism among 4 resistant and 4 susceptible peanut lines for rust and late leaf spot diseases. These transferable markers will benefit peanut genome research by not only providing additional DNA markers for population genetic analyses, but also allowing comparative mapping to be possible between peanut and sorghum - a possible monocot-dicot comparison.
\end{abstract}

Keywords: Arachis Hypogea; Transferability; EST-SSR; Polymorphism

\section{Introduction}

Peanut (Arachis hypogaea L.) is an important oilseed crop and it has acquired prominence because of its economic importance as well as its nutritional value. It is the third major oilseed crop in the world next only to soybean and cotton. A. hypogaea is believed to have originnated in the Southern Bolivia to Northern Argentina region of South America. The present day cultivated peanut is an allotetraploid $(2 n=4 x=40)$ while most of wild relatives are diploid $(2 n=2 x=20)$ in nature. The yield of the peanut crop has been very low due to biotic and abiotic stresses and the varietal improvement in peanut has been difficult due to the limited knowledge on the inheritance of important traits and lack of proper understanding of genetic diversity and population structure. Molecular markers have become an important tool in crop breeding programs for dissecting loci controlling complex traits: genetic diversity among accession and evolutionary conservation studies can be done. However, application of molecular markers in peanut crop improvement has been relatively lagging behind chiefly owing to limited knowledge of genome and seldom molecular variations revealed by RFLP [1], RAPD [2] and

${ }^{*}$ Corresponding author. isozyme markers systems [3]. Indeed, there is an urgent need to focus efforts on a systematic and comprehensive examination of the germplasm accessions available in the peanut employing robust marker types such as SSRs to reveal polymorphism at the molecular level [4]. Simple Sequence Repeats (SSRs) or microsatellites have become one of the most widely preferred molecular marker systems for genetic analysis for their advantages compared to other molecular markers: high reproducibility, high polymorphism, being multi-allelic, co-dominant, higher relative abundance and extensive genome coverage are some of the advantages envisaged with SSRs [5]. Previous studies in peanut have shown that SSR markers could detect more polymorphism than other molecular markers like RFLPs [6], AFLPs [7] and RAPDs [8,9].

The de novo development of SSRs markers is a costly and time-consuming endeavor [5,10], as it involves approaches, such as genomic library construction, enrichment and screening which are laborious and time consuming: this reduces the general utility of this marker system [11] and also dramatically discounts the advantages [12]. The progress of development or discovery of new marker types has shifted from anonymous DNA fragments to gene-based markers, also called as functional markers. Gene based markers are more powerful 
than others for breeding applications and allele discovery [13]. ESTs are presently used on a large scale for the systematic development of gene-based SSR and SNP markers. EST-SSR markers have been developed for a number of plant species, such as pigeon pea [14], grape [15], rice [16], durum wheat [17], rye [18], barley [19], ryegrass [20], wheat [21], peanut [22] and cotton [23]. EST-SSRs are advantageous over genomic SSRs, as they can be obtained from public EST databases.and transferable across taxonomic barriers [24]. A putative function can be deduced for the EST-SSRs as they represent ESTs, they serve as gene-tagged markers and can be directly associated with an expressed gene: this offers linking with putative qualitative or quantitative trait locus alleles. Thus, EST-SSR markers are superior and more informative compared to anonymous markers [25]. Comparative genetic analysis has shown that different plant species often share orthologous genes for very similar functions [26] and gene contents and gene orders among different plant species could be highly conserved [27,28].

As EST-SSR markers are derived from expressed genes, they are more conserved and have a higher level of transferability to related species. Study of transferability of markers has been attempted in several plant species across different taxa $[14,15,19,29-32]$ as well as in peanut $[12,33,34]$. However, the conserved nature of EST-SSRs may also limit their degree of polymorphism. The feasibility of utilizing EST-SSRs from monocots in dicots has been investigated. Plant genes display significant conservation between the monocots and dicots, thus, theoretical possibility of transferability from monocots to dicots is a possibility [35]. Requiring more concerted efforts in using modern genomic tools, peanut genome research has made less progress [36,37]. Thus, one of the pressing needs in peanut genomic research is to take advantage of progress made in the well characterized other crops. About 25\% SSRs [38] and 34\% EST-SSRs [39] transferability from soybean to peanut has been reported [40]. A $20 \%$ transferability of EST-SSRs from Medicago to peanut has been reported. In this study, we focused on analyzing the utility of EST-SSR markers from sorghum (monocot) to peanut (dicot) experimentally. Sorghum is considered to be model grass genome where genetic study has been done at good pace [41] and its genome sequencing is completed [42], and hence it could be good source of transferable markers especially the gene-based markers.

\section{Materials and Methods}

Total DNA from sorghum cultivar E36-1 and a set of four resistant and four rust and leaf spot diseases susceptible peanut cultivars (Table 1) was isolated following CTAB protocol of Murry and Thompson (1980) [43] with suitable modifications. The genomic DNA was used as the template for all PCR amplifications. Sorghum EST-SSRs (SbEST-SSRs) developed at IABT, UAS, Dharwad and synthesized from Sigma-Aldrich pvt. Ltd, USA, were screened for amplification of peanut DNA using optimized PCR reaction mixture and touchdown PCR Profiles. PCR optimization was done using three different programs of "Touchdown" PCR [44] with base annealing temperature ranges of $55^{\circ} \mathrm{C}-50^{\circ} \mathrm{C}, 60^{\circ} \mathrm{C}$ $55^{\circ} \mathrm{C}$, and $65^{\circ} \mathrm{C}-60^{\circ} \mathrm{C}$. The primers were classified into three groups based on annealing temperature range required by them to produce sharp bands without much of spurious products. In the initial annealing steps, the annealing temperature was decreased by one centigrade after two subsequent cycles for first 10 cycles. Products were thereafter amplified for 30 cycles at the appropriate optimum annealing temperature with a final extension of $20 \mathrm{~min}$. Reaction mixtures of $10 \mu \mathrm{l}$ containing $10 \mathrm{mM}$ Tris- $\mathrm{HCl}$ ( $\mathrm{pH} 8.8$ ), $50 \mathrm{mM} \mathrm{KCl}, 10 \mathrm{mM} \mathrm{MgCl} 2,2.5 \mathrm{mM}$ of each of dNTPs, $20 \mathrm{pM}$ each of forward and reverse primers, $50 \eta \mathrm{g}$ of genomic DNA and 2.5U Taq DNA polymerase (Fermentas) was used for PCR amplification.

Transference is defined as the positive amplification of a PCR band of the expected size [45]. SbEST-SSR primers amplified during primer screening of peanut were also used for comparing amplification patterns (size and

Table 1. Transferable markers were checked for their ability to detect polymorphism in following eight groundnut cultivars.

\begin{tabular}{ccccc}
\hline SI No & Genotypes & Rust & Late leaf spot & Phenotypes \\
\hline 1 & LSVT-I-2003-1 & 2 & 2 & $\mathrm{R}$ \\
2 & ISK-I-2004-4 & 2 & 1 & $\mathrm{R}$ \\
3 & IVT-I-2005-5 & 2 & 1 & $\mathrm{R}$ \\
4 & GPBD-4 & 3 & 3 & $\mathrm{R}$ \\
5 & JL-24 & 7 & 8 & $\mathrm{~S}$ \\
6 & TMV-2 & 7 & $\mathrm{~S}$ \\
7 & TAG-24 & 7 & $\mathrm{~S}$ \\
8 & TG-26 & 7 & 8 & $\mathrm{~S}$ \\
\hline
\end{tabular}

Cultivars with a 1 - 3 disease score were designated as resistant and a 4 - 9 score as susceptible according to Pande and Rao (2001). 
number of bands) to further confirm orthology or transferability by carrying out amplification in both sorghum and peanut DNA using optimized PCR reaction mixture and touchdown PCR profiles. The transferable SbESTSSRs were then tested for their ability to detect polymorphism in a set of four resistant and four susceptible breeding lines/cultivars (Table 1) for rust and late leaf spot diseases of peanut and the ones showing polymerphic banding pattern on 4\% PAGE gel were considered as polymorphic markers.

\section{Results}

\subsection{Screening of SbEST-SSRs and Comparison of Amplification Pattern between Sorghum and Peanut and Their Polymorphism in Peanut Cultivars}

Out of 411 sorghum EST-SSR primer pairs tested, 161 (39\%) were amplifiable in peanut (Table 1) showing clear sharp bands but other primers gave smear with light bands or did not amplify under three Touch Down (TD)PCR profile conditions (Figure 1). The remaining primer pairs either recorded no amplification products or produced a number of faint bands indicating non-specific amplifications. Out of 161 amplified primers 16 amplified at $65^{\circ} \mathrm{C}-60^{\circ} \mathrm{C}, 95$ at $60^{\circ} \mathrm{C}-55^{\circ} \mathrm{C}$ and 61 at $55^{\circ} \mathrm{C}-$ $50^{\circ} \mathrm{C}$ TD-PCR temperature ranges (Table 2). These amplifiable markers implied that $39 \%$ of primer-binding sites were conserved between sorghum and peanut genomes. These primer pairs produced clear PCR bands and the majority of primers produced multiple bands. The number of bands amplified by each SbEST-SSR primer pairs varied from 1 to 16 on $4 \%$ polyacrylamide gel stained following silver staining procedure (Figure 2). Further, comparison of the kind of amplification pattern between sorghum and peanut crop species showed similar banding pattern for many of the SbEST-SSRs; however, it varied in some of the cases (Figure 3) and the difference was in number of bands amplified, which were more or less in either of the crops. Of the161 EST-SSRs $18 \%$ were found polymorphic on $4 \%$ polyacrylamide gel.

\subsection{Structural Analysis and Annotations of SbEST-SSRs}

Among these conserved SbEST-SSRs, 91 (56\%) dinucleotide repeat motifs appeared to be the most abundant type, followed by $61(37.2 \%)$ trinucleotides, $8(6.3 \%)$ consisting of complex nucleotide repeats and then one tetranucleotide $(0.5 \%)$ repeats (Table 3$)$. Among these repeats $\mathrm{TC} / \mathrm{CT}$ were $35, \mathrm{AG} / \mathrm{GA}$ were $28, \mathrm{AGC} / \mathrm{CAG} /$ GCA were 17 and AGC/CAG/GCA were 16. The composition of repeat motifs of transferred SbEST-SSRs is presented in Table 4. Of 161 SbEST-SSR markers, 24 (14\%) detected polymorphism on $4 \%$ PAGE among 8 accessions of peanut consisting of equal number of resistant and susceptible accessions (Figure 2). Twenty four polymorphic SbEST-SSRs consisted of 17 (18.7\%) dinucleotide repeats, $6(9.8 \%)$ trinucleotide repeats and one $(1.2 \%)$ consisted of complex repeat motifs. These results indicate that there is some kind of correlation between polymorphism and repeat number. The distribution of transferred SSRs' positions was found highest in 5'ESTs with 67, then 32 in 3 'ESTs and 62 in other ESTs. Among these the SSRs from 3'ESTs were more polymorphic (21.9\%) than 5'ESTs (13.4\%) and others (12.9\%) (Table 4).

Annotation for the common SbEST-SSRs was performed using the GenBank databases and BLASTX tool with an expectation value of 1e-5 or better. Eighty three $(52 \%)$ of the common ESTs were annotated using BLASTX and are listed in Table 5. Most of annotated SbESTs were related to metabolism, photosynthesis, signal transduction, growth, and transportation across membranes, stress and defense. The remaining SbESTs when searched for putative functions resulted in no hits $(2.5 \%)$,

Table 2. Screening results for 411 SbEST-SSR primers on groundnut genotypes: total number and the percentages of primer pairs amplified at three different Touch Down PCR profiles.

\begin{tabular}{ccccccc}
\hline Sl. No & PCR & No of Primers & Rate & $\mathbf{6 5}^{\circ} \mathbf{C}-\mathbf{6 0}^{\circ} \mathbf{C}$ & $\mathbf{6 0}^{\circ} \mathbf{C}-\mathbf{5 5}{ }^{\circ} \mathbf{C}$ & $\mathbf{5 5}^{\circ} \mathbf{C}-\mathbf{5 0}^{\circ} \mathbf{C}$ \\
\hline 1 & Amplified & 161 & $39 \%$ & $19(12 \%)$ & $81(50 \%)$ & $61(38 \%)$ \\
2 & Unamplified & 250 & $61 \%$ & - & - & - \\
& Total & 411 & $\mathbf{1 0 0 \%}$ & - & - & - \\
\hline
\end{tabular}

Table 3. Structural details of repeat motifs in transferable SbEST-SSRs and polymorphism revealed by them in groundnut.

\begin{tabular}{ccccc}
\hline Type & Dinucleotides & Trinucleotides & Tetranucleotide & Complex \\
\hline Amplified & 91 & 61 & 1 & 8 \\
Per cent Amplification & 56.5 & 37.9 & 0.6 & 5 \\
Polymorphic & 17 & 6 & 0 & 1 \\
Per cent Polymorphic & 18.7 & 9.8 & 0 & 1.2 \\
\hline
\end{tabular}


Table 4. Distribution of repeats location or SSRs positions in ESTs of transferable SbEST-SSRs.

\begin{tabular}{cccc}
\hline Type & 3'ESTs & 5'ESTs & Others \\
\hline Amplified & 32 & 67 & 62 \\
Per cent Amplification & 19.9 & 41.6 & 38.5 \\
Polymorphic & 7 & 9 & 8 \\
Per cent Polymorphic & 21.9 & 13.4 & 12.9 \\
\hline
\end{tabular}

Table 5. Description of SbEST-SSR markers transferred to groundnut: NCBI accession ID, repeat motifs, their ability to detect polymorphism among groundnut accessions and their functional annotation by BLASTX.

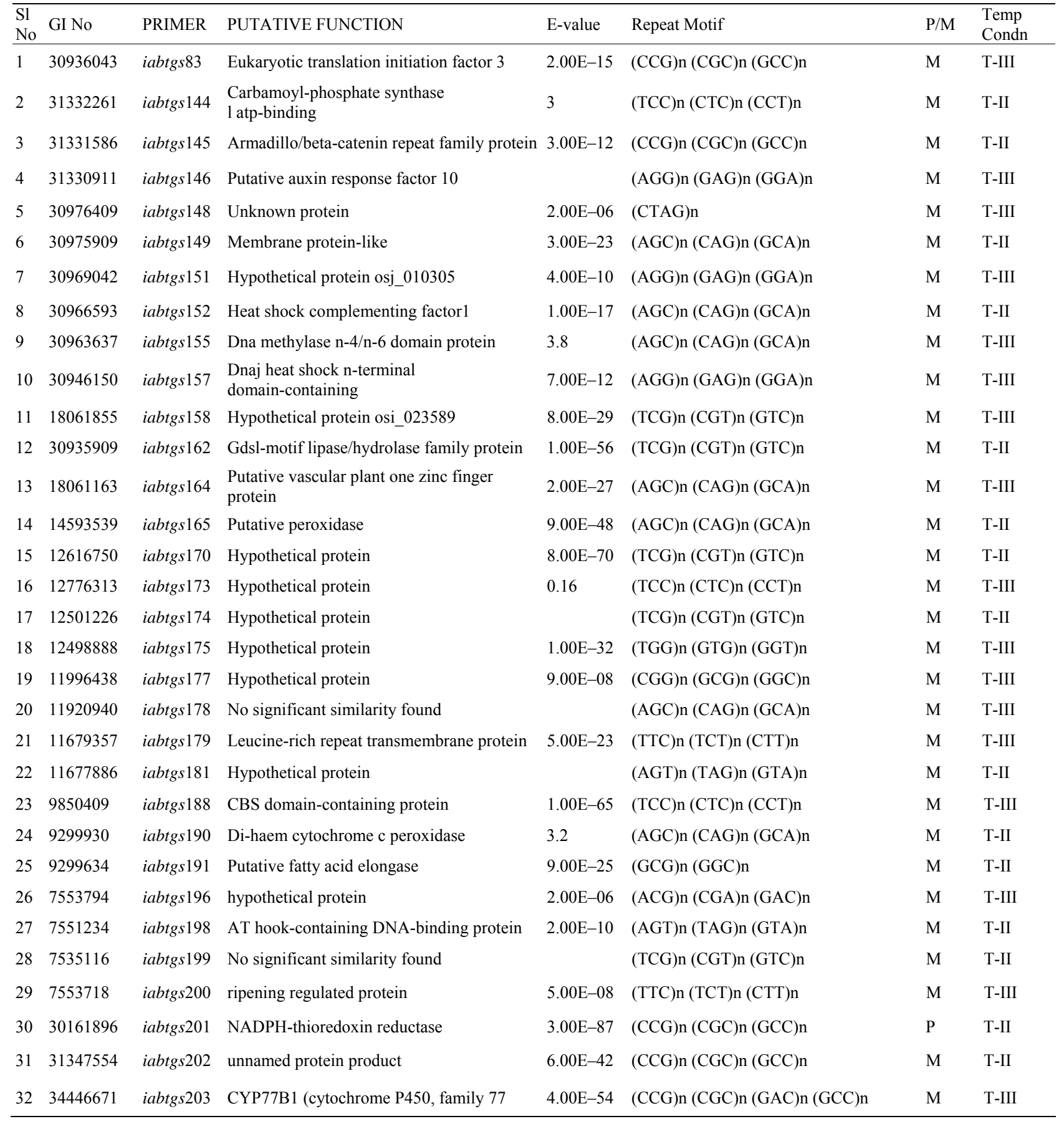




\section{Continued}

\begin{tabular}{|c|c|c|c|c|c|c|c|}
\hline 33 & 30977482 & iabtgs 205 & transporter-related & $1.00 \mathrm{E}-35$ & $(\mathrm{AGG}) \mathrm{n}(\mathrm{GAG}) \mathrm{n}(\mathrm{GGA}) \mathrm{n}$ & $\mathrm{M}$ & T-II \\
\hline 34 & 34512855 & iabtgs 207 & hypothetical protein OsI_000407 & $6.00 \mathrm{E}-75$ & (TTG)n (TGT)n (GTT)n & M & T-III \\
\hline 35 & 7536147 & iabtgs 208 & putative xyloglucan endotransglycosylase & $9.00 \mathrm{E}-46$ & $(\mathrm{CGG}) \mathrm{n}(\mathrm{GCG}) \mathrm{n}(\mathrm{GGC}) \mathrm{n}$ & M & T-II \\
\hline 36 & 30950922 & iabtgs 209 & 3-ketoacyl-CoA synthase & $1.00 \mathrm{E}-51$ & $(\mathrm{AGC}) \mathrm{n}(\mathrm{CAG}) \mathrm{n}(\mathrm{GCA}) \mathrm{n}$ & M & T-III \\
\hline 37 & 45949294 & iabtgs 210 & ETHYLENE-INSENSITIVE3-like 1 protein & $3.00 \mathrm{E}-45$ & $(\mathrm{AGC}) \mathrm{n}(\mathrm{CAG}) \mathrm{n}(\mathrm{GCA}) \mathrm{n}$ & M & T-II \\
\hline 38 & 30968428 & iabtgs 220 & putative copper chaperone & $5.00 \mathrm{E}-27$ & (TC)n (CT)n /(TGC)n (CTG)n (GCT)n & M & T-II \\
\hline 39 & 57812929 & iabtgs 221 & ACR4 (ACT REPEAT 4) & $2.00 \mathrm{E}-26$ & (ACT)n (TAC)n (CTA)n/(TC)n (CT)n & $\mathrm{P}$ & T-II \\
\hline 40 & 37753412 & iabtgs 222 & harpin inducing protein & $1.00 \mathrm{E}-09$ & (TGC)n (CTG)n (GCT)n & $\mathrm{P}$ & T-II \\
\hline 41 & 9296509 & iabtgs 223 & dehydration responsive element binding & $2.00 \mathrm{E}-22$ & $(\mathrm{ACC}) \mathrm{n}(\mathrm{CAC}) \mathrm{n}(\mathrm{CCA}) \mathrm{n}$ & M & T-III \\
\hline 42 & 61099098 & iabtgs 225 & hypothetical protein OsJ_024137 & $2.00 \mathrm{E}-17$ & $(\mathrm{CGG}) \mathrm{n}(\mathrm{GCG}) \mathrm{n}(\mathrm{GGC}) \mathrm{n}$ & M & T-II \\
\hline 43 & 45993487 & iabtgs 226 & glycoside hydrolase family 28 protein & $1.00 \mathrm{E}-56$ & (TGC)n (CTG)n (GCT)n & $\mathrm{P}$ & T-I \\
\hline 44 & 9305233 & iabtgs 227 & unknown protein & & $(\mathrm{AGC}) \mathrm{n}(\mathrm{CAG}) \mathrm{n}(\mathrm{GCA}) \mathrm{n}$ & $\mathrm{M}$ & T-I \\
\hline 45 & 34510491 & iabtgs 228 & Hypothetical protein & & (CGG)n (GCG)n (GGC)n & $\mathrm{P}$ & T-II \\
\hline 46 & 9303289 & iabtgs 229 & Nitrate induced NOI protein & $1.00 \mathrm{E}-40$ & $(\mathrm{CCG}) \mathrm{n}(\mathrm{CGC}) \mathrm{n}(\mathrm{GCC}) \mathrm{n} /(\mathrm{AG}) \mathrm{n}(\mathrm{GA}) \mathrm{n}$ & $\mathrm{M}$ & T-II \\
\hline 47 & 18052712 & iabtgs 230 & ZIM motif-containing protein & 0.12 & $(\mathrm{AGC}) \mathrm{n}(\mathrm{CAG}) \mathrm{n}(\mathrm{GCA}) \mathrm{n}$ & $\mathrm{M}$ & T-I \\
\hline 48 & 33110457 & iabtgs 231 & hypothetical protein & $1.00 \mathrm{E}-19$ & (TCG)n (CGT)n (GTC)n & $\mathrm{P}$ & T-I \\
\hline 49 & 45957262 & iabtgs 234 & leaf senescence related protein-like & $9.00 \mathrm{E}-78$ & $(\mathrm{AGC}) \mathrm{n}(\mathrm{CAG}) \mathrm{n}(\mathrm{GCA}) \mathrm{n}$ & $\mathrm{M}$ & T-II \\
\hline 50 & 14089228 & iabtgs 235 & Ethylene receptor & $3.00 \mathrm{E}-61$ & $(\mathrm{CCG}) \mathrm{n}(\mathrm{CGC}) \mathrm{n}(\mathrm{GCC}) \mathrm{n}$ & M & T-I \\
\hline 51 & 57808251 & iabtgs 237 & $\mathrm{CHCH}$ & $2.00 \mathrm{E}-12$ & $(\mathrm{CCG}) \mathrm{n}(\mathrm{CGC}) \mathrm{n}(\mathrm{GCC}) \mathrm{n}$ & M & T-III \\
\hline 52 & 45965553 & iabtgs 242 & Cytochrome P450 71E1 & $3.00 \mathrm{E}-85$ & (ACG)n (CGA)n (GAC)n & M & T-II \\
\hline 53 & 30163708 & iabtgs 244 & phosphoglycerate mutase-like protein & $8.00 \mathrm{E}-52$ & $(\mathrm{AGC}) \mathrm{n}(\mathrm{CAG}) \mathrm{n}(\mathrm{GCA}) \mathrm{n}$ & M & T-II \\
\hline 54 & 34443079 & iabtgs 250 & No significant similarity found. & & $(\mathrm{AGG}) \mathrm{n}(\mathrm{GAG}) \mathrm{n}(\mathrm{GGA}) \mathrm{n}$ & $\mathrm{M}$ & T-III \\
\hline 55 & 30968371 & iabtgs 251 & putative copper chaperone & $5.00 \mathrm{E}-27$ & $(\mathrm{AGC}) \mathrm{n}(\mathrm{CAG}) \mathrm{n}(\mathrm{GCA}) \mathrm{n}$ & $\mathrm{M}$ & T-II \\
\hline 56 & 34443334 & iabtgs 259 & No significant similarity found & & $(\mathrm{AAG}) \mathrm{n}(\mathrm{AGA}) \mathrm{n}(\mathrm{GAA}) \mathrm{n}$ & $\mathrm{M}$ & T-II \\
\hline 57 & 45990071 & iabtgs 260 & Diadenosine 5',5"'-P1 tetraP hydrolase & $1.00 \mathrm{E}-88$ & $(\mathrm{CCG}) \mathrm{n}(\mathrm{CGC}) \mathrm{n}(\mathrm{GCC}) \mathrm{n}$ & $\mathrm{M}$ & T-III \\
\hline 58 & 34517721 & iabtgs 263 & putative inositol-3-phosphate synthase & $3.00 \mathrm{E}-30$ & $(\mathrm{AGC}) \mathrm{n}(\mathrm{CAG}) \mathrm{n}(\mathrm{GCA}) \mathrm{n}$ & $\mathrm{M}$ & T-III \\
\hline 59 & 30945913 & iabtgs 264 & chloroplast O2-evolving enhancer protein 1 & $8.00 \mathrm{E}-68$ & (ACG)n (CGA)n (GAC)n & M & T-III \\
\hline 60 & 45968979 & iabtgs 269 & EREBP transcription factor & $9.00 \mathrm{E}-46$ & $(\mathrm{AGG}) \mathrm{n}(\mathrm{GAG}) \mathrm{n}(\mathrm{GGA}) \mathrm{n}$ & $\mathrm{M}$ & T-II \\
\hline 61 & 9303947 & iabtgs 274 & hypothetical protein & $3.00 \mathrm{E}-25$ & $(\mathrm{ACC}) \mathrm{n}(\mathrm{CAC}) \mathrm{n}(\mathrm{CCA}) \mathrm{n}$ & $\mathrm{M}$ & T-II \\
\hline 62 & 33108470 & iabtgs 280 & PyroP-dependent phosphofructokinase & $2.00 \mathrm{E}-22$ & $(\mathrm{AAG}) \mathrm{n}(\mathrm{AGA}) \mathrm{n}(\mathrm{GAA}) \mathrm{n} /(\mathrm{AG}) \mathrm{n}(\mathrm{GA}) \mathrm{n}$ & M & T-II \\
\hline 63 & 34440910 & iabtgs 281 & putative 4,5-DOPA dioxygenase extradiol & $1.00 \mathrm{E}-37$ & $(\mathrm{AGG}) \mathrm{n}(\mathrm{GAG}) \mathrm{n}(\mathrm{GGA}) \mathrm{n}$ & M & T-II \\
\hline 64 & 45975671 & iabtgs 282 & Plastocyanin precursor & $7.00 \mathrm{E}-19$ & (TCC)n (CTC)n (CCT)n & $\mathrm{M}$ & T-II \\
\hline 65 & 31332334 & iabtgs 287 & No significant similarity found. & & $(\mathrm{TC}) \mathrm{n}(\mathrm{CT}) \mathrm{n}$ & $\mathrm{M}$ & T-II \\
\hline 66 & 37711087 & iabtgs 289 & Cyclic beta 1-2 glucan synthetase & 3 & $(\mathrm{AG}) \mathrm{n}(\mathrm{GA}) \mathrm{n}$ & M & T-II \\
\hline 67 & 18062597 & iabtgs301 & No significant similarity found & & (AT)n (TA)n & M & T-I \\
\hline 68 & 30946136 & iabtgs304 & unknown protein & $8.00 \mathrm{E}-09$ & (TC)n (CT)n & $\mathrm{M}$ & T-III \\
\hline 69 & 30164571 & iabtgs305 & No significant similarity found & & $(\mathrm{TC}) \mathrm{n}(\mathrm{CT}) \mathrm{n}$ & M & T-III \\
\hline 70 & 7535895 & iabtgs307 & Mitogen activated protein kinase 6 & $2.00 \mathrm{E}-06$ & (TC)n (CT)n & $\mathrm{P}$ & T-II \\
\hline 71 & 11921286 & iabtgs309 & AMP-binding protein & $1.00 \mathrm{E}-35$ & $(\mathrm{AG}) \mathrm{n}(\mathrm{GA}) \mathrm{n}$ & $\mathrm{M}$ & T-III \\
\hline 72 & 9851429 & iabtgs 310 & unknown & & $(\mathrm{AC}) \mathrm{n}(\mathrm{CA}) \mathrm{n}$ & $\mathrm{P}$ & T-III \\
\hline 73 & 30939660 & iabtgs314 & putative polyprotein & $9.00 \mathrm{E}-14$ & (TG)n (GT)n & $\mathrm{P}$ & T-III \\
\hline 74 & 30964510 & iabtgs322 & hypothetical protein & $3.00 \mathrm{E}-15$ & $(\mathrm{AG}) \mathrm{n}(\mathrm{TC}) \mathrm{n}$ & M & T-II \\
\hline 75 & 11922775 & iabtgs 323 & hypothetical protein & $2.00 \mathrm{E}-26$ & (AT)n (TA)n & $\mathrm{M}$ & T-II \\
\hline
\end{tabular}




\begin{tabular}{|c|c|c|c|c|c|c|c|}
\hline \multicolumn{8}{|c|}{ Continued } \\
\hline 76 & 31329611 & iabtgs324 & hypothetical protein OsJ_018717 & $8.00 \mathrm{E}-58$ & $(\mathrm{AG}) \mathrm{n}(\mathrm{GA}) \mathrm{n}$ & M & T-II \\
\hline 77 & 18052335 & iabtgs327 & proline-rich spliceosome-associated factor & $8.00 \mathrm{E}-37$ & (AT)n (TA)n & M & T-II \\
\hline 78 & 31383744 & iabtgs 340 & $\begin{array}{l}\text { cytochrome P450 monooxygenase } \\
\text { CYP77B5 }\end{array}$ & $7.00 \mathrm{E}-52$ & $(\mathrm{AG}) \mathrm{n}(\mathrm{GA}) \mathrm{n}$ & $\mathrm{P}$ & T-II \\
\hline 79 & 57821918 & iabtgs 341 & Chloroplast phytoene synthase 1 & $9.00 \mathrm{E}-38$ & $(\mathrm{AG}) \mathrm{n}(\mathrm{TG}) \mathrm{n}(\mathrm{GA}) \mathrm{n}(\mathrm{GT}) \mathrm{n}$ & M & T-III \\
\hline 80 & 57807306 & iabtgs342 & Serine/threonine-protein kinase MHK & $3.00 \mathrm{E}-07$ & $(\mathrm{AC}) \mathrm{n}(\mathrm{CA}) \mathrm{n}$ & M & T-III \\
\hline 81 & 45993419 & iabtgs343 & APX4_SOLLC L-ascorbate peroxidase & $8.00 \mathrm{E}-33$ & $(\mathrm{AG}) \mathrm{n}(\mathrm{GA}) \mathrm{n}$ & $\mathrm{P}$ & T-II \\
\hline 82 & 37759348 & iabtgs 345 & hypothetical protein HEAR0860 & 8.7 & $(\mathrm{AG}) \mathrm{n}(\mathrm{GA}) \mathrm{n}$ & M & T-III \\
\hline 83 & 45969660 & iabtgs346 & E3 ubiquitin ligase & $9.00 \mathrm{E}-06$ & (TC)n (CT)n & M & T-III \\
\hline 84 & 34509854 & iabtgs349 & $\begin{array}{l}\text { LHT2 (LYSINE HISTIDINE } \\
\text { TRANSPORTER 2) }\end{array}$ & & (TG)n (GT)n & $\mathrm{P}$ & T-II \\
\hline 85 & 34517131 & iabtgs350 & auxin efflux carrier family protein & $2.00 \mathrm{E}-40$ & (TC)n (CT)n & M & T-III \\
\hline 86 & 37753820 & iabtgs352 & Urease accessory protein UreD & 2.3 & (AT)n (TA)n & $\mathrm{P}$ & T-II \\
\hline 87 & 37711166 & iabtgs 353 & COG0583: Transcriptional regulator & 3.2 & $(\mathrm{TC}) \mathrm{n}(\mathrm{CT}) \mathrm{n}$ & M & T-II \\
\hline 88 & 37706443 & iabtgs355 & $\begin{array}{l}\text { isoprenylcysteine carboxyl } \\
\text { methyltransferase }\end{array}$ & 9.3 & (TG)n (GT)n & M & T-II \\
\hline 89 & 37705823 & iabtgs356 & No significant similarity found & & (TC)n (CT)n & M & T-III \\
\hline 90 & 37705032 & iabtgs357 & SJCHGC01974 protein & 2.1 & $(\mathrm{AC}) \mathrm{n}(\mathrm{CA}) \mathrm{n}$ & M & T-III \\
\hline 91 & 34509495 & iabtgs359 & hypothetical protein & $3.00 \mathrm{E}-05$ & $(\mathrm{AG}) \mathrm{n}(\mathrm{GA}) \mathrm{n}$ & M & T-II \\
\hline 92 & 34442668 & iabtgs 360 & hydroxycinnamoyl transferase & $3.00 \mathrm{E}-31$ & $(\mathrm{AG}) \mathrm{n}(\mathrm{GA}) \mathrm{n}$ & M & T-II \\
\hline 93 & 33110250 & iabtgs362 & $\mathrm{NADH}$-ubiquinone oxidoreductase-protein & $3.00 \mathrm{E}-27$ & $(\mathrm{AG}) \mathrm{n}(\mathrm{GA}) \mathrm{n}$ & M & T-III \\
\hline 94 & 34443764 & iabtgs363 & No significant similarity found & & $(\mathrm{AC}) \mathrm{n}(\mathrm{CA}) \mathrm{n}$ & $\mathrm{P}$ & T-I \\
\hline 95 & 34440481 & iabtgs364 & hypothetical protein & $2.00 \mathrm{E}-09$ & $(\mathrm{AC}) \mathrm{n}(\mathrm{CA}) \mathrm{n}$ & M & T-III \\
\hline 96 & 33110768 & iabtgs366 & putative shrunken seed protein & 0.78 & $(\mathrm{TC}) \mathrm{n}(\mathrm{CT}) \mathrm{n}$ & M & T-II \\
\hline 97 & 33107765 & iabtgs367 & No significant similarity found & & (AT)n (TA)n & M & T-III \\
\hline 98 & 31330527 & iabtgs369 & No significant similarity found & & $(\mathrm{TC}) \mathrm{n}(\mathrm{CT}) \mathrm{n}$ & $\mathrm{P}$ & T-II \\
\hline 99 & 30979643 & iabtgs371 & cation efflux system protein , RNA helicase & 4.4 & (TG)n (GT)n & M & T-II \\
\hline 100 & 30976566 & iabtgs375 & hypothetical protein & & (AT)n (TA)n & M & T-III \\
\hline 101 & 30974675 & iabtgs377 & Plant viral-response family protein & $5.00 \mathrm{E}-06$ & $(\mathrm{CG}) \mathrm{n}$ & M & T-III \\
\hline 102 & 30974381 & iabtgs378 & putative Myb-like DNA-binding protein & $1.00 \mathrm{E}-07$ & $(\mathrm{AG}) \mathrm{n}(\mathrm{GA}) \mathrm{n}$ & M & T-II \\
\hline 103 & 30947756 & iabtgs 381 & hypothetical protein & 1.6 & $(\mathrm{AG}) \mathrm{n}(\mathrm{GA}) \mathrm{n}$ & M & T-II \\
\hline 104 & 30964406 & iabtgs382 & Glycyl-tRNA synthetase & 0.59 & $(\mathrm{AG}) \mathrm{n}(\mathrm{GA}) \mathrm{n}$ & M & T-III \\
\hline 105 & 30966299 & iabtgs 383 & GHMP kinase family protein & $7.00 \mathrm{E}-39$ & $(\mathrm{AG}) \mathrm{n}(\mathrm{GA}) \mathrm{n}$ & M & T-II \\
\hline 106 & 30937801 & iabtgs384 & putative membrane protein & 0.9 & (TC)n (CT)n & M & T-II \\
\hline 107 & 18052318 & iabtgs385 & hypothetical protein Os01g0260800 & 0.002 & (AT)n (TA)n & $\mathrm{P}$ & T-I \\
\hline 108 & 30936750 & iabtgs386 & heat shock protein & $2.00 \mathrm{E}-33$ & (TG)n (GT)n & M & T-II \\
\hline 109 & 18065756 & iabtgs387 & No significant similarity found & & (TG)n (GT)n & M & T-II \\
\hline 110 & 18060131 & iabtgs397 & hypothetical protein & & $(\mathrm{AG}) \mathrm{n}(\mathrm{GA}) \mathrm{n}$ & $P$ & T-II \\
\hline 111 & 18051780 & iabtgs399 & No significant similarity found & & (TC)n (CT)n & M & T-II \\
\hline 112 & 14513091 & iabtgs 407 & hypothetical protein & $1.00 \mathrm{E}-27$ & (TG)n (GT)n & M & T-II \\
\hline 113 & 13587956 & iabtgs 408 & hypothetical protein & $4.00 \mathrm{E}-05$ & $(\mathrm{TC}) \mathrm{n}(\mathrm{CT}) \mathrm{n}$ & M & T-III \\
\hline 114 & 13469439 & iabtgs 410 & No significant similarity found & & $(\mathrm{AG}) \mathrm{n}(\mathrm{GA}) \mathrm{n}$ & M & T-II \\
\hline 115 & 11678719 & iabtgs411 & putative laccase & $3.00 \mathrm{E}-67$ & (TC)n (CT)n & M & T-II \\
\hline 116 & 12776058 & iabtgs 416 & hypothetical protein & $2.00 \mathrm{E}-44$ & (AT)n (TA)n & M & T-III \\
\hline 117 & 11409368 & iabtgs 421 & hypothetical protein & 2.2 & $(\mathrm{AC}) \mathrm{n}(\mathrm{CA}) \mathrm{n}$ & M & T-II \\
\hline 118 & 9850860 & iabtgs 425 & WRKY transcription factor 16 & 2.2 & (AT)n (TA)n & M & T-II \\
\hline
\end{tabular}


Continued

\begin{tabular}{|c|c|c|c|c|c|c|}
\hline 1199305649 & iabtgs 429 & No significant similarity found & & (AT)n (TA)n & M & T-III \\
\hline 1207536347 & iabtgs 430 & calcium channel & 8.5 & (TC)n (CT)n & M & T-III \\
\hline 12130973885 & iabtgs 439 & Adenosine 5'-phpsphosulfate reductase 6 & $2.00 \mathrm{E}-76$ & $(\mathrm{TC}) \mathrm{n}(\mathrm{CT}) \mathrm{n}$ & M & T-III \\
\hline 12257815251 & iabtgs 440 & Endoxyloglucan transferase & & (TG)n (GT)n & $\mathrm{P}$ & T-II \\
\hline 12330160959 & iabtgs 441 & shaggy-like kinase etha (OSKetha) & $9.00 \mathrm{E}-50$ & $(\mathrm{CCG}) \mathrm{n}(\mathrm{CGC}) \mathrm{n}$ & M & T-I \\
\hline 12431332570 & iabtgs 445 & para-hydroxybenzoate-polyprenyltransferase & 3.6 & (TC)n (CT)n & M & T-III \\
\hline 12533109119 & iabtgs 447 & NADP dependent maleic enzyme & $1.00 \mathrm{E}-14$ & $(\mathrm{AG}) \mathrm{n}(\mathrm{GA}) \mathrm{n}$ & $\mathrm{P}$ & T-III \\
\hline 1267659319 & iabtgs 449 & No significant similarity found & & (TG)n (GT)n & M & T-III \\
\hline 12711922518 & iabtgs 450 & transcription factor MybS3 & $3.00 \mathrm{E}-53$ & $(\mathrm{TC}) \mathrm{n}(\mathrm{CT}) \mathrm{n}$ & $\mathrm{P}$ & T-III \\
\hline 12830974889 & iabtgs 455 & Nucellin like aspartic protease & $9.00 \mathrm{E}-43$ & $(\mathrm{AG}) \mathrm{n}(\mathrm{GA}) \mathrm{n}$ & M & T-II \\
\hline 12933109611 & iabtgs 456 & unknown protein & $8.00 \mathrm{E}-06$ & (TC)n (CT)n & M & T-I \\
\hline 13017886525 & iabtgs 457 & hypothetical protein & & $(\mathrm{AG}) \mathrm{n}(\mathrm{GA}) \mathrm{n}$ & M & T-II \\
\hline 13161099192 & iabtgs 458 & No significant similarity found & & $(\mathrm{AC}) \mathrm{n}(\mathrm{CA}) \mathrm{n}$ & M & T-I \\
\hline 13233108498 & iabtgs 460 & hypothetical protein & 0.65 & $(\mathrm{AG}) \mathrm{n}(\mathrm{GA}) \mathrm{n}$ & M & T-III \\
\hline 13333108027 & iabtgs 464 & hypothetical protein & & (TC)n (CT)n & M & T-III \\
\hline 13434442937 & iabtgs 470 & ATTIC21/CIA5/PIC1/(chloropast Import) & $3.00 \mathrm{E}-20$ & (TG)n (GT)n & $P$ & T-II \\
\hline 13530937618 & iabtgs 472 & No significant similarity found & & (TGC)n (CTG)n (GCT)n /(TG)n (GT)n & M & T-I \\
\hline 13630942400 & iabtgs 473 & ribosomal-protein-alanine acetyltransferase & 3.2 & (TC)n (CT)n & M & T-II \\
\hline 13718070466 & iabtgs 478 & VHS2 protein & $1.00 \mathrm{E}-04$ & (AT)n (TA)n & M & T-I \\
\hline 13830939303 & iabtgs 484 & Alpha tubulin & $9.00 \mathrm{E}-08$ & $(\mathrm{TC}) \mathrm{n}(\mathrm{CT}) \mathrm{n}$ & M & T-I \\
\hline 13912497850 & iabtgs 487 & hypothetical protein & 5.1 & (AT)n (AC)n /(TA)n (TC)n (CA)n (CT)n & M & T-II \\
\hline 14031385392 & iabtgs 488 & Protease inhibitor/seed storage/LTP family & $1.00 \mathrm{E}-15$ & $(\mathrm{AG}) \mathrm{n}(\mathrm{GA}) \mathrm{n}$ & M & T-II \\
\hline 14130975546 & iabtgs491 & quinone-oxidoreductase QR1 & $6.00 \mathrm{E}-07$ & (TC)n (CT)n & M & T-II \\
\hline 14245960926 & iabtgs 492 & No significant similarity found & & (TC)n (CT)n & M & T-II \\
\hline 14330952757 & iabtgs 493 & hypothetical protein & $3.00 \mathrm{E}-07$ & (TG)n (GT)n & $P$ & T-III \\
\hline 14412618782 & iabtgs 496 & putative receptor-like kinase & $7.00 \mathrm{E}-10$ & (TC)n (CT)n & M & T-III \\
\hline 1459304578 & iabtgs 499 & retrotransposon protein & 0.2 & $(\mathrm{AC}) \mathrm{n}(\mathrm{CA}) \mathrm{n}$ & M & T-II \\
\hline 14618066228 & iabtgs500 & Serine/threonine-protein kinase SSN3 & 8.5 & (TG)n (GT)n & M & T-I \\
\hline 14712775763 & iabtgs502 & putative membrane protein & $4.00 \mathrm{E}-07$ & (TC)n (CT)n & M & T-III \\
\hline 14861115436 & iabtgs504 & No significant similarity found & & (AT)n (TA)n & M & T-I \\
\hline 1498088843 & iabtgs505 & $\begin{array}{l}\text { peptidase } \mathrm{C} 14 \text {, caspase catalytic subunit } \\
\text { p20 }\end{array}$ & 8.7 & $(\mathrm{AG}) \mathrm{n}(\mathrm{GA}) \mathrm{n}$ & M & T-III \\
\hline 15045961441 & iabtgs507 & No significant similarity found & & $(\mathrm{AG}) \mathrm{n}(\mathrm{GA}) \mathrm{n}$ & M & T-III \\
\hline 15133109939 & iabtgs 510 & prolylcarboxypeptidase-like protein & 0.089 & (TC)n (CT)n & $\mathrm{P}$ & T-I \\
\hline 15211678708 & iabtgs 512 & hypothetical protein & 0.002 & $(\mathrm{AGC}) \mathrm{n}(\mathrm{CAG}) \mathrm{n}(\mathrm{GCA}) \mathrm{n} /(\mathrm{AG}) \mathrm{n}(\mathrm{GA}) \mathrm{n}$ & M & T-II \\
\hline 1535043542 & iabtgs514 & hypothetical protein & & $(\mathrm{AT}) \mathrm{n}$ & M & T-II \\
\hline 15434445779 & iabtgs516 & $\begin{array}{l}\text { Chitin-inducible gibberllin-responsive } \\
\text { protein }\end{array}$ & $4.00 \mathrm{E}-40$ & $(\mathrm{AG}) \mathrm{n}(\mathrm{GA}) \mathrm{n}$ & M & T-II \\
\hline 15533108255 & iabtgs 517 & No significant similarity found & & (TC)n (CT)n & M & T-I \\
\hline 15630973999 & iabtgs 518 & hypothetical protein & $2.00 \mathrm{E}-15$ & (TC)n (CT)n & M & T-III \\
\hline 15757821918 & iabtgs520 & No significant similarity found & & & M & T-II \\
\hline 15834515133 & iabtgs528 & Ubiquitin-conjugating enzyme like & $3.00 \mathrm{E}-12$ & (TC)n (CT)n & M & T-II \\
\hline 15933108946 & iabtgs529 & hypothetical protein & & (TC)n (CT)n & $P$ & T-II \\
\hline 1609852958 & iabtgs 530 & No significant similarity found & & (AT)n (TA)n & M & T-I \\
\hline 16145988690 & iabtgs531 & RISBZ4 & & $(\mathrm{CGG}) \mathrm{n}(\mathrm{GCG}) \mathrm{n}(\mathrm{GGC}) \mathrm{n} /(\mathrm{AG}) \mathrm{n}(\mathrm{GA}) \mathrm{n}$ & M & T-I \\
\hline
\end{tabular}

-T-I, T-II and T-III represent the three Touchdown PCR thermal profiles viz. $60^{\circ} \mathrm{C}-55^{\circ} \mathrm{C}, 55^{\circ} \mathrm{C}-50^{\circ} \mathrm{C}$ and $50^{\circ} \mathrm{C}-45^{\circ} \mathrm{C}$ respectively. 


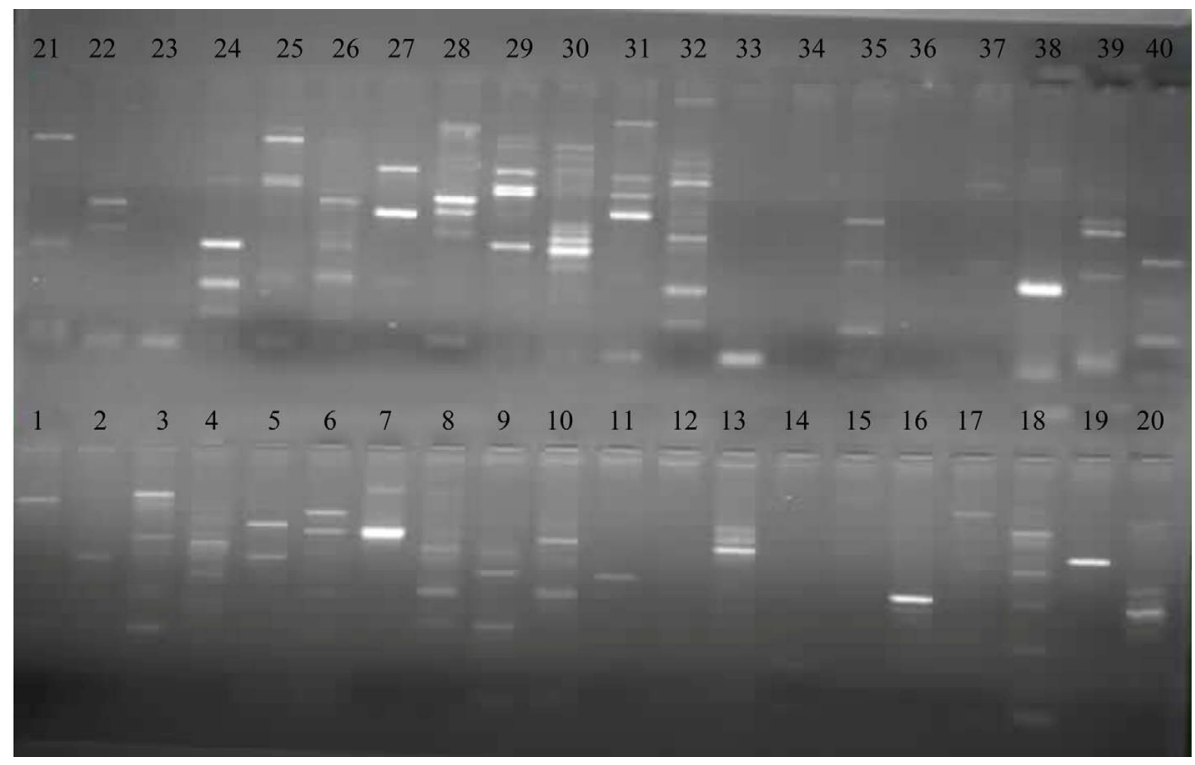

Figure 1. Amplification pattern generated by sorghum EST-SSR (SbEST-SSRs) during primer screening in groundnut using genomic DNA. 1-40: Amplification products of SbEST-SSR primers in groundnut during primer screening.

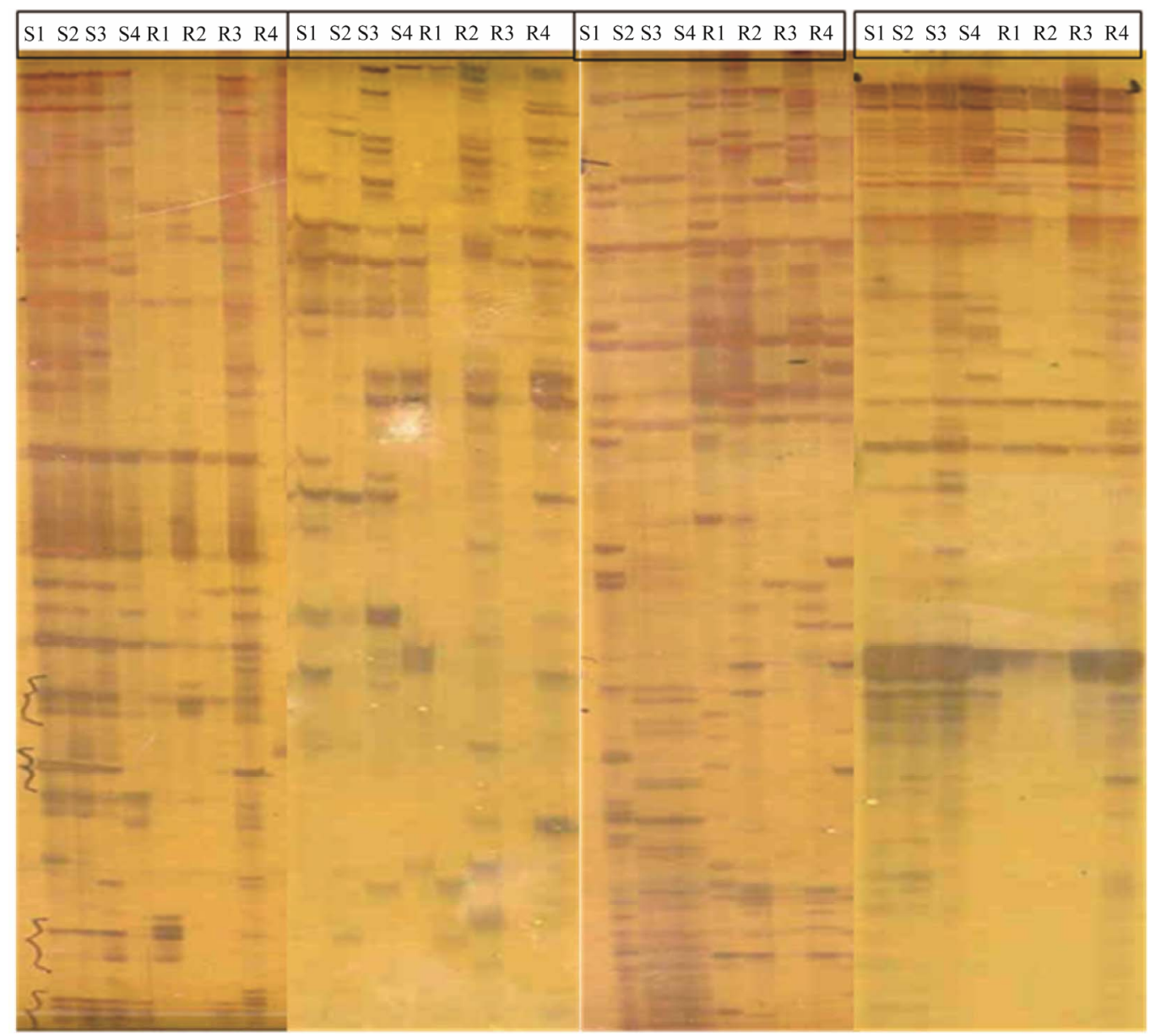

Figure 2. The figure is a composite of multiple polyacrylamide gels which illustrates the multiple and polymorphic bands generated using the SbEST-SSR primers in eight groundnut accessions. S1 - S4: Four rust and leafspot susceptible genotypes JL-24, TMV-2, TAG-24 and TG-26; R1-R4: Four rust and leafspot susceptible genotypes LSVT-I-2003-1, ISK-I-2004-4, IVT-I-2005-5and GPBD-4. 


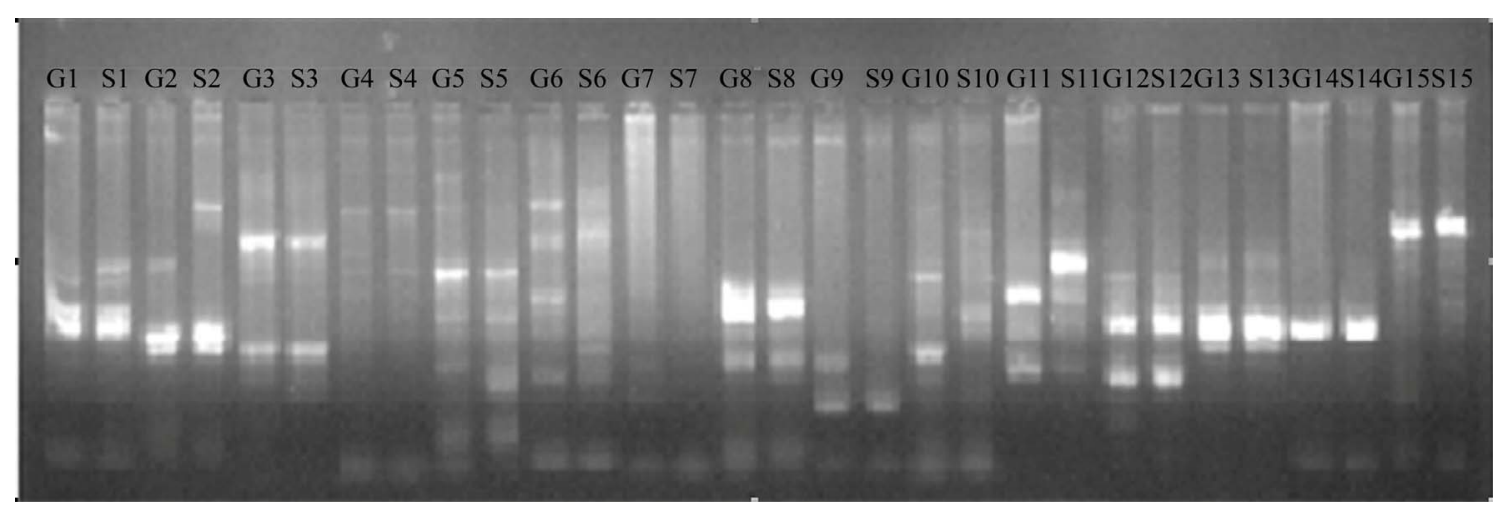

Figure 3. Comparison of amplification patterns in both groundnut and sorghum using SbEST-SSR markers. G1 S1 - G15 S15: Comparison of PCR amplification in groundnut (G) and sorghum (S) with SbEST-SSRs.

no significant homology $(9.3 \%)$, or hypothetical proteins (37\%) (Table 1). In major cases of ESTs putative functions matched both monocots (rice, maize) and dicots (Arabidopsis, soybean) indicating their common sharing.

\section{Discussion}

Despite the tremendous diversity, plant geneticists have found that plants exhibit extensive conservation of both gene content and gene order [27]. Sequence similarity of the barley ESTs with 379,944 ESTs of the two model dicot species, Arabidopsis and Medicago suggested theoretical transferability of barley markers into dicot species although at low frequency [24] and EST-SSR by virtue of the sequence conservation of the transcribed regions of the genome are more likely to function in distantly related species than SSR primer pair derived from genomic libraries.

In the present study 161 out of 411 SbEST-SSRs amplified in peanut. So, about $39 \%$ transferability or conservation of SSR motifs and flanking sequences found between sorghum (monocot) and peanut (dicot). The orthology was further confirmed by comparing amplifycation pattern (number of amplification products and size) in both sorghum and peanut genomes. Majority of them had similar amplification pattern but a few showed extra bands with common ones either in sorghum or peanut which may be due to duplications, insertions or deletion mutations during course of evolution which diverged 150 million years ago [46]. Similar bands amplified regardless of phylogenetic distances are an important feature of EST-SSR markers which are transferred across species or even genera [29]. But one of the concerns is alleles of identical size with different numbers of repeats within the SSR (size homoplasy)) observed in most of studies, suggesting a need for caution when interpreting alleles of identical size found using cross-amplified SSRs based on band migration in the absence of DNA sequences. So knowledge of DNA sequence is essential before SSR loci can be meaningfully used to address applied and evolu- tionary questions. Majority of SbEST-SSRs produced multiple bands (range 1 - 16) which is a common feature reported in most of the studies involving transferability of EST-SSRs.

Transferability of SbEST-SSRs in the present study is more $(39 \%)$ compared to study of He et al. [47] using soybean genomic SSRs $(25 \%)$ in peanut. But the polymorphism detection rate in this study $(18 \%)$ is less compared to latter study (28\%). This illustrate that EST-SSRs are more transferable across species or distant taxa and are less efficient in polymorphism detection than genomic SSRs as they are derived from transcribed regions of genome which are conserved across species. However, transferability (39\%) in this study is less compared to the study of Gao et al. (2003) [12]. In which 69\% transferability from wheat (monocot system) to soybean (dicot system) was observed, but percent transferability or conserved EST-SSRs from wheat to rice, maize and soybean in the same study $43 \%$. With regard to developing microsatellite markers, 3'-sequences yielded more polymorphic markers $(22.9 \%)$ than 5 -ESTs $(13.4 \%)$ did. This result is not unexpected as during the process of cDNA generation (poly $\mathrm{T}$ priming) there is a preferential selection of untranslated regions (UTR) within 3'-ESTs, therefore are more variable than 5 '-ESTs. In the distribution of SSR motifs, dinucleotides were found more common than tri, tetra or complex nucleotide repeats in transferred SbEST-SSRs or new gene based markers accounting for $56 \%, 37.2 \%, 0.5 \%$ and $5 \%$ respectively and also SbEST-SSRs with dinucleotide repeats detected more polymerphism $(18.7 \%)$ than tri $(9.8 \%$, ) and complex nucleotide $(1.2 \%)$ repeat motifs. Dinucletotides were also found to detect higher polymorphism than others, which was observed in some the previous studies [48]. This shows that there seems to be some correlation between repeat number and polymorphism.

The transferable SbEST-SSRs subjected to BlastX with an e-value more than or equal to $1 \mathrm{E}-5$ as a significant homology, could annotate putative functions for 
$52 \%$ of the common ESTs (Table 3). Most of annotated SbESTs were related to basic functions of plant cells such as metabolism, photosynthesis, signal transduction, transcription, growth, and transportation across membranes, stress and defense. The remaining SbESTs search for putative functions resulted in poor hits $(2.5 \%)$ no significant homology $(9.3 \%)$ or hypothetical proteins $(37 \%)$. These may represent transcriptomes which are yet to be characterized for their putative functions. In major cases of ESTs putative functions matched both monocots (rice, maize) and dicots (Arabidopsis, soybean) suggesting that these are highly conserved across plant species mainly encoding for basic functions. Thus annotations of transferred SbEST-SSRs help to explore the potential utility of the EST-SSR loci for comparative mapping in peanut. Functional EST-SSRs exhibiting sequence similarity to genes with a range of functions could be used directly in determining putative traits. For example, ESTsequences of iabtgs366 and iabtgs269 showed a strong homology to putative shrunken seed protein and EREBP transcription factor, which is involved in stress tolerance respectively. This potential will make them a valuable source of new genic SSR markers so called "perfect" genetic markers.

Thus, by using transferability technique it was possible to develop a set of new gene based markers for peanut crop using genomic resources of sorghum that will be useful for different genetic studies in peanut. In this study, we could demonstrate the feasibility of utilizing EST-SSRs from monocots in dicots as plant genes display significant conservation even after the long period of independent evolution.

\section{Acknowledgements}

Authors are thankful to the Indo-US Agricultural Knowledge Initiative and Department of Biotechnology (DBT) of Government of India for supporting research in authors' (BF) laboratory.

\section{REFERENCES}

[1] T. Halward, H. T. Stalker and G. Kochert, "Development of an RFLP Linkage Map in Peanut Species," Theoretical and Applied Genetics, Vol. 87, No. 3, 1993, pp. 379-384. doi:10.1007/BF01184927

[2] M. D. Burrow, C. E. Simpson, A. H. Paterson and J. L. Starr, "Identification of Peanut (Arachis hypogaea L.) RAPD Markers Diagnostic of Root-Knot Nematode (Meloidigyne arenaria (Neal) Chitwood) Resistance," Molecular Breeding, Vol. 2, No. 4, 1996, pp. 368-379. doi:10.1007/BF00437915

[3] G. Lacks and H. Stalker, "Isozyme Analyses of Arachis Species and Interspecific Hybrids," Peanut Science, Vol. 20, No. 2, 1993, pp. 76-81. doi:10.3146/i0095-3679-20-2-3
[4] A. K. Singh, J. Smartt, C. E. Simpson and S. N. Raina, "DNA Markers in Cultivated Peanut (Arachis hypogaea L.)," BMC Plant Biology, Vol. 3, 1998, pp. 3-10.

[5] L. Zane, L. Bargelloni and T. Patarnello, "Strategies for Microsatellite Isolation: A Review," Molecular Ecology, Vol. 11, No. 1, 2002, pp. 1-16. doi:10.1046/j.0962-1083.2001.01418.x

[6] M. A. Gimenes, A. A. Hoshino, A. V. G. Barbosa, D. A. Palmieri and C. R. Lope, "Characterization and Transferability of Microsatellite Markers of the Cultivated Peanut (A. hypogaea)," BMC Plant Biology, Vol. 7, 2007, p. 9. http://www.biomedcentral.com/bmcplantbiol. doi:10.1186/1471-2229-7-9

[7] G. He and C. Prakash, "Evaluation of Genetic Relationship among Botanical Varieties of Cultivated Peanut (Arachis hypogaea L.) Using AFLP Markers," Genetic Resources and Crop Evolution, Vol. 48, No. 4, 2001, pp. 347-352. doi:10.1023/A:1012019600318

[8] S. L. Dwivedi, S. Gurtu, S. Chandra, W. Yuejin and S. N. Nigam, "Assessment of Genetic Diversity among Selected Groundnut Germplasm. I: RAPD Analysis," Plant Breeding, Vol. 120, No. 4, 2001, pp. 345-349. doi:10.1046/j.1439-0523.2001.00613.x

[9] V. Subramanian, S. Gurtu, R. C. Nageswara Rao and S. N. Nigam, "Identification of DNA Polymorphism in Cultivated Groundnut Using Random Amplified Polymorphic DNA (RAPD) Assay," Genome, Vol. 43, No. 4, 2000, pp. 656-660. doi: 10.1139/g00-034

[10] J. Squirrell, P. M. Hollingsworth, M. Woodhead, J. Russell, A. J. Lowe and M. Gibby, "How Much Effort Is Required to Isolate Nuclear Microsatellites from Plants?" Molecular Ecology, Vol. 12, 2003, pp. 1339-1348. doi:10.1046/j.1365-294X.2003.01825.X

[11] J. R. Ellies and J. M. Burke, "EST-SSRs as a Resource for Population Genetic Analyses," Heredity, Vol. 99, No. 2, 2007, pp. 125-132. doi:10.1038/sj.hdy.6801001

[12] L. Gao, J. Tang, H. Li and J. Jia, "Analysis of Microsatellites in Major Crops Assessed by Computational and Experimental Approaches," Molecular Breeding, Vol. 12, No. 3, 2003, pp. 245-261.

[13] Jia and Bonierbale, "Validation of Conserved Orthologous Markers, A Proposal for Extension of Commissioned Research in Subprogram 2 'Comparative Genomics' Cluster 2: Marker Development Generation Challenge Program," 2005.

[14] N. L. Raju, B. N. Gnanesh and R. Varshney, "The First Set of EST Resource for Gene Discovery and Marker Development in Pigeonpea (Cajanus cajan L.)," BMC Plant Biology, Vol. 10, 2010, pp. 45-67. doi:10.1186/1471-2229-10-45

[15] G. M. Cordeiro, R. Casu, C. L. Mcintyre, J. M. Manners and R. J. Henry, "Microsatellite Markers from Sugarcane (Saccharum spp.) ESTs cross Transferable to Erianthus and Sorghum," Plant Science, Vol. 160, No. 6, 2001, pp. 1115-1123. doi:10.1016/S0168-9452(01)00365-X

[16] S. Temnykh, G. Declerck, A. Lukashova, L. Lipovich, S. Cartinhour and S. Mccouch, "Computational and Experimental Analysis of Microsatellites in rice (O. sativa L.): Frequency, Length Variation, Transposon Associa- 
tions, and Genetic Marker Potential," Genome Research, Vol. 11, No. 8, 2001, pp. 1441-1452.

doi:10.1101/gr.184001

[17] I. Eujayl, M. E. Sorrells, P. Wolters, M. Baum and W. Powell, "Isolation of EST-Derived Microsatellite Markers for Genotyping the A and B Genomes of Wheat," Theoretical and Applied Genetics, Vol. 104, No. 2-3, 2002, pp. 399-407. doi:10.1007/s001220100738

[18] B. Hackauf and P. Wehling, "Identification of Microsatellite Polymorphisms in an Expressed Portion of the Rye Genome," Plant Breeding, Vol. 121, No. 1, 2002, pp. 1725. doi:10.1046/j.1439-0523.2002.00649.x

[19] T. Thiel, W. Michalek, K. Varsheny and A. Graner, "Exploiting EST Databases for the Development of cDNA Derived Microsatellite Markers in Barley (Hordeum vulgare L.)," Theoretical and Applied Genetics, Vol. 106, No. 3, 2003, pp. 411-422.

[20] M. J. Faville, A. C. Vecchies, M. Schreiber, M. C. Drayton, L. J. Hughes, E. S. Jones, K. M. Guthridge, K. F. Smith, T. Sawbridge, G. C. Spangenberg, G. T. Bryan and J. W. Forster, "Functionally Associated Molecular Genetic Marker Map Construction in Perennial Ryegrass (Lolium perenne L.)," Theoretical and Applied Genetics, Vol. 110, No. 1, 2004, pp. 12-32. doi:10.1007/s00122-004-1785-7

[21] J. H. Peng and N. L. Lapitan, "Characterization of ESTDerived Microsatellites in the Wheat Genome and Development of eSSR Markers," Functional \& Integrative Genomics, Vol. 5, No. 2, 2005, pp. 80-96. doi:10.1007/s10142-004-0128-8

[22] G. Q. Song, M. J. Li, H. Xiao, X. J. Wang, R. H. Tang, H. Xia, et al., "EST Sequencing and SSR Marker Development from Cultivated Peanut (Arachis hypogaea L.)," Electronic Journal of Biotechnology, Vol. 13, No. 3, 2010, pp. 1-9. doi: $10.2225 /$ vol13

[23] Z. Han, C. Wang, X. Song, W. Guo, J. Gou, C. Li, X. Chen and T. Zhang, "Characteristics, Development and Mapping of Gossypium hirsutum Derived EST-SSRs in Allotetraploid Cotton," Theoretical and Applied Genetics, Vol. 112, No. 3, 2006, pp. 430-439. doi:10.1007/s00122-005-0142-9

[24] R. K. Varshney, R. Sigmund, A. Borner, V. Korzun, N. Stein and M. E. Sorrells, "Interspecific Transferability and Comparative Mapping of Barley EST-SSR Markers in Wheat, Rye and Rice," Plant Science, Vol. 168, No. 1, 2005, pp. 195-202. doi:10.1016/j.plantsci.2004.08.001

[25] J. R. Andersen and T. Lubberstedt, "Functional Markers in Plants," Trends in Plant Science, Vol. 8, No. 11, 2003, pp. 554-560. doi:10.1016/j.tplants.2003.09.010

[26] C. A. Fatokun, D. I. Menacio-Hautea, D. Danesh and N. D. Young, "Evidence for Orthologous Seed Weight Genes in Cowpea and Mung Bean Based upon RFLP Mapping," Genetics, Vol. 132, 1992, pp. 841-846.

[27] J. L. Bennetzen and M. Freeling, "Grasses as a Single Genetic System: Genome Composition, Colinearity and Compatibility," Trends in Genetics, Vol. 9, No. 8, 1993, pp. 259-261. doi:10.1016/0168-9525(93)90001-X

[28] M. D. Gale and K. M. Devos, "Comparative Genetics in the Grasses," Proceedings of the National Academy of
Sciences of the USA, Vol. 95, 1998, pp. 1971-1974.

[29] V. Decroocq, M. G. Fave, L. Hagen, L. Bordenave and S. Decroocq, "Development and Transferability of Apricot and Grape EST Microsatellite Markers across Taxa," Theoretical and Applied Genetics, Vol. 106, No. 5, 2003, pp. 912-922.

[30] M. Woodhead, J. Russell, J. Squirrell, P. M. Hollingsworth, L. Cardle, L. Ramsay, M. Gibby and W. Powell, "Development of EST-SSRs from the Alpine Lady-Fern, Athyrium Distentifolium," Molecular Ecology Notes, Vol. 3, No. 2, 2003, pp. 287-290. doi:10.1046/j.1471-8286.2003.00427.x

[31] M. V. Gutierrez, M. C. Vaz Patto and T. Huguet, "CrossSpecies Amplification of Medicago Truncatula Microsatellites across Three Major Pulse Crops," Theoretical and Applied Genetics, Vol. 110, No. 7, 2005, pp. 1210-1217. doi:10.1007/s00122-005-1951-6

[32] X. Jia, Y. Shi, Y. Song, G. Wang, T. Wang and Y. Li, "Development of EST-SSR in Foxtail Millet (Setaria italica)," Genetic Resources and Crop Evolution, Vol. 54, No. 2, 2007, pp. 233-236. doi:10.1007/s10722-006-9139-8

[33] X. Liang, X. Chen, Y. Hong, H. Liu, G. Zhou, S. Li and B. Guo, "Utility of EST-Derived SSR in Cultivated Peanut (Arachis hypogaea L.) and Arachis Wild Species," BMC Plant Biology, Vol. 9, 2009, p. 35. doi:10.1186/1471-2229-9-35

[34] B. Gautami, K. Ravi, M. L. Narasu, D. A. Hoisington and R. K. Varshney, "Novel Set of Peanut SSR Markers for Germplasm Analysis and Interspecific Transferability," International Journal of Integrative Biology, Vol. 7, No. 2, 2009, pp. 100-106.

[35] R. K. Varshney, A. Graner and M. E. Sorrells, "Genic Microsatellite Markers in Plants: Features and Applications," Trends in Biotechnology, Vol. 23, No. 1, 2005, pp. 48-54. doi:10.1016/j.tibtech.2004.11.005

[36] M. Luo, P. Dang, B. Z. Guo, G. He, C. C. Holbrook, M. G. Bausher and R. D. Lee, "Generation of Expressed Sequence Tags (ESTs) for Gene Discovery and Marker Development in Cultivated Peanut," Crop Science, Vol. 45, No. 1, 2005, pp. 346-353. doi: $10.2135 /$ cropsci2005.0346

[37] M. Luo, P. Dang, M. G. Bausher, C. C. Holbrook, R. D. Lee, R. E. Lynch and B. Z. Guo, "Identification of Transcripts Involved in Resistance Response to Leaf Spot Disease Caused by Cercosporidium personatum in Peanut (Arachis hypogaea)," Phytopathology, Vol. 95, No. 4, 2005, pp. 381-387. doi:10.1094/PHYTO-95-0381

[38] G. He, R. H. Meng, H. Gao, B. Guo, G. Gao, M. Newman, R. N. Pittman and C. S. Prakash, "Simple Sequence Repeat Markers for Botanical Varieties of Cultivated Peanut (Arachis hypogaea L.)," Euphytical, Vol. 142, No. 1, 2005, pp. 131-136. doi:10.1007/s10681-005-1043-3

[39] G. H. He, R. H. Meng, M. Newman, G. Q. Gao, R. N. Pittman and C. S. Prakash, "Microsatellites as DNA Markers in Cultivated Peanut (Arachis hypogaea L.)," BMC Plant Biology, Vol. 3, 2003, p. 3. doi:10.1186/1471-2229-3-3

[40] M. L. Wang, N. L. Barkley, R. Dean, C. Holbrook and R. 
N. Pittman, "Transfer of Medicago EST-SSRs to Peanut for Germplasm Evaluation and Cross-Species Cloning," Annual American Peanut Research \& Education Society Proceedings, Vol. 7, 2004, pp. 6-12.

[41] J. E. Mullet, R. R. Klein and P. E. Klein, "Sorghum bicolor-An Important Species for Comparative Grass Genomics and a Source of Beneficial Genes for Agriculture," Current Opinion in Plant Biology, Vol. 5, No. 2, 2002, pp. 118-121. doi:10.1016/S1369-5266(02)00232-7

[42] A. H. Paterson, et al., "The Sorghum bicolor Genome and the Diversification of Grasses," Nature, Vol. 457, No. 7229, 2009, pp. 551-556. doi:10.1038/nature07723

[43] M. G. Murray and W. F. Thompson, "Rapid Isolation of High Molecular Weight Plant DNA," Nucleic Acids Research, Vol. 8, 1980, pp. 4321-4325. doi:10.1093/nar/8.19.4321

[44] R. H. Don, P. T. Cox, B. J. Wainwright, K. Baker and J. S. Mattick, "Touchdown PCR to Circumvent Spurious Priming during Gene Amplification," Nucleic Acids Research,
Vol. 19, 1991, p. 4008. doi:10.1093/nar/19.14.4008

[45] S. C. Gonza'lez-Marti'nez, N. C. Wheeler Elhan Ersoz, D. C. Nelson and D. B. Neal, "Association Genetics in Pinus taeda L. I. Wood Property Traits," Genetics, Vol. 175, 2007, pp. 399-409.

[46] W. L. Crepet and G. D. Feldman, "The Earliest Remains of Grasses in the Fossil Record," American Journal of Botany, Vol. 78, No. 7, 1991, pp. 1010-1014. doi: $10.2307 / 2445181$

[47] G. H. He, F. E. Woullard, I. Marong and B. Z. Guo, "Transferability of Soybean SSR Markers in Peanut (Arachis hypogaea L.)," Peanut Science, Vol. 33, No. 1, 2006, pp. 22-28. doi:10.3146/0095-3679(2006)33[22:TOSSMI]2.0.CO;2

[48] J. K. Yu, A. E. Strand and B. G. Milligan, "EST-Derived SSR Markers for Comparative Mapping in Wheat and Rice," Molecular Genetics and Genomics, Vol. 271, No. 6, 2004, pp. 742-751. doi:10.1007/s00438-004-1027-3 